\title{
Further Results on Stability Analysis of Discrete-Time Markov Jump Linear Systems with Time-Varying Delay and Partly Known Transition Probabilities
}

\author{
Xingang Zhao, Jianda Han, and Yiwen Zhao \\ State Key Laboratory of Robotics, Shenyang Institute of Automation, Chinese Academy of Sciences, Shenyang 110016, China \\ Correspondence should be addressed to Xingang Zhao; zhaoxingang2014@163.com
}

Received 8 August 2014; Revised 23 October 2014; Accepted 23 October 2014

Academic Editor: Quanxin Zhu

Copyright (c) 2015 Xingang Zhao et al. This is an open access article distributed under the Creative Commons Attribution License, which permits unrestricted use, distribution, and reproduction in any medium, provided the original work is properly cited.

\begin{abstract}
This paper is concerned with the stability analysis of discrete-time Markov jump linear systems (MJLSs) with time-varying delay and partly known transition probabilities. The time delay is varying between lower and upper bounds, and the partly known transition probabilities cover the cases of known, uncertain with known lower and upper bounds, and completely unknown, which is more general than the existing result. Via constructing an appropriate Lyapunov function and employing a new technique to separate Lyapunov variables from unknown transition probabilities, a novel stability criterion is obtained in the framework of linear matrix inequality. A numerical example is given to show the effectiveness of the proposed approach.
\end{abstract}

\section{Introduction}

Markov jump linear systems (MJLSs), a class of stochastic systems, have been widely applied in manufacturing systems, power systems, aerospace systems, and networked control systems. This is due to its ability to model many practical systems with abrupt random changes in their structures and parameters. Regarding the details on the basic theory of MJLSs, for example, controllability, observability, stability, stabilisability, and optimal control, and so forth, please refer to $[1-8]$ and the references therein.

Time delay, unavoidable in many practical systems, can degrade the closed loop system performance or even lead the resulting system to be unstable. In recent years, therefore, the time delay problem has been widely studied and a great number of research results concerning this point exist in the references for linear system; see, for example, [9-13] and so on. Regarding the study of MJLSs with time delay, mean square stochastic stability conditions are presented in [7] and exponential stability conditions are proposed in [8]. However, these results are delay independent. To make full use of the available information on the size of delays, delay-dependent results are more attractive. Employing a model transformation, delay-dependent stability conditions for MJLSs with constant delay are proposed in [14]. To derive less conservative results, this idea was further improved by $[15,16]$. Instead of using model transformation or bounding for cross terms, zero equations are utilized by [17]. Considering the fact that time delay for practical system is time varying, based on the free weighting matrix technique, [18] proposes a less conservative stability criterion for MJLSs with time-varying delays. By introducing some improved integral equalities, a new stability condition for MJLSs with timevarying delay is proposed in [19]. Motivated by above results, [20] proposes improved stochastic stability conditions for MJLSs with interval time delay. Regarding the stability analysis of Markovian jump neural systems with mixed delay, sample data control, impulse control, and exponential stability are investigated in [21-27]. With resorting to new technique to deal with the time delay, based on a LyapunovKrasovskii functional and the stochastic analysis theory, some novel sufficient conditions are established in the framework of linear matrix inequalities [22-27]. It deserves to note that all above results are based on transition probabilities which are known. Practically, it is hard or costly to obtain the complete knowledge on the transition probabilities [28, 29]. A typical example can be found in networked control systems (NCSs). Due to the complexity of the network environment, 
the variation of delays could be vague and random, which leads to the fact that all or part of the elements in the expected transition probabilities matrix are probably hard or expensive to obtain [28-32]. To be consistent with the actual situation, transition probabilities in [28-32] are assumed to be known or unknown. With this assumption, stability analysis of MJLSs with time-varying delay is investigated in [32]. However, in these results, when transition probabilities are unknown with known lower and upper bounds, they are treated as completely unknown, which may cause conservativeness. Moreover, the technique to separate Lyapunov variables and unknown transition probabilities in [32] still leaves some room for further improvement.

Motivated by the above observations, this paper is devoted to the stability analysis of discrete-time MJLSs with time-varying delay and partly known transition probabilities. The boundary information of time-varying delay is made full use of and the partly known transition probabilities include the cases of known, uncertain with known lower and upper bounds, and completely unknown, which is more general than the existing result. Via constructing an appropriate Lyapunov-Krasovskii functional, combining the property of transition probabilities and free weighted matrix technique, a novel stability criterion is obtained in the framework of linear matrix inequality. A numerical example is utilized to show the effectiveness of the proposed approach.

The rest of the paper is organized as follows. Section 2 gives the problem description, in Section 3, a novel theorem about delay range dependent stability for systems with more general transition probabilities is derived by using Lyapunov function method, Section 4 illustrates the efficiency of the proposed method, and Section 5 concludes this paper.

Notation. $R^{n}$ denotes the $n$ dimensional Euclidean space and the notation $P>0(P \geq 0)$ means that $P$ is real symmetric and positive definite (semidefinite). $N^{+}$represents the set of positive integers. In symmetric block matrices or complex matrix expressions, we use an asterisk $(*)$ to represent a term that is induced by symmetry and diag... stands for a blockdiagonal matrix. $I$ and 0 represent, respectively, the identity matrix and zero matrix.

\section{System Description and Preliminaries}

Consider the following class of discrete-time Markov jump linear systems:

$$
\begin{gathered}
x(k+1)=A\left(r_{k}\right) x(k)+A_{d}\left(r_{k}\right) x(k-d(k)), \\
x(k)=\phi(k), \quad k=-d_{M},-d_{M}+1, \ldots, 0,
\end{gathered}
$$

where $x(k) \in R^{n}$ is the state vector. The time delay is considered to be time-varying with known lower and upper bounds, namely, $0 \leq d_{m} \leq d_{k} \leq d_{M} \cdot r_{k}(k \geq 0)$ is a discretetime homogeneous Markov chain, which takes values in a finite set $\mathscr{I}=\{1,2, \ldots, N\}$ with the following mode transition probabilities:

$$
\operatorname{Pr}(r(k+1)=j \mid r(k)=i)=\pi_{i j},
$$

where $\pi_{i j} \geq 0$ and $\sum_{j=1}^{N} \pi_{i j}=1$.
Considering the fact that transition probabilities are hard to be measured exactly, they are assumed to be known, unknown with known lower and upper bounds, and completely unknown in this context [31]. To present the considered transition probabilities clearly, an example of partly known transition probability matrix is given in the following:

$$
\Pi=\left[\begin{array}{ccccc}
\pi_{11} & ? & \pi_{13} & \cdots & \pi_{1 N} \\
? & ? & \alpha & \cdots & \pi_{2 N} \\
\beta & ? & ? & \cdots & \pi_{3 N} \\
\vdots & \cdots & \cdots & \ddots & \vdots \\
\pi_{N 1} & \pi_{N 2} & ? & \cdots & \pi_{N N}
\end{array}\right]
$$

where "?" denotes that the corresponding element is completely unknown, $\alpha$ and $\beta(\underline{\alpha}<\alpha<\bar{\alpha}, \underline{\beta}<\beta<\bar{\beta})$ represent unknown elements with known bounds, and $\pi_{i j}$ represents the exactly known elements. Subsequently, all transition probabilities in the above matrix can be classified by the following two sets:

$$
\begin{gathered}
\mathscr{I}_{k}^{i} \triangleq\left\{j: \underline{\pi}_{i j} \leq \pi_{i j} \leq \bar{\pi}_{i j}\right\}, \\
\mathscr{I}_{u k}^{i} \triangleq\left\{j: j \notin \mathscr{I}_{k}^{i}\right\} .
\end{gathered}
$$

Remark 1. With the fact that known transition probabilities can be treated as equal lower and upper bounds, that is to say, $\underline{\pi}_{i j}=\bar{\pi}_{i j}=\pi_{i j}$, the first set, $\mathscr{J}_{k}^{i}$, includes not only unknown transition probabilities with known bounds but also completely known case.

Before proceeding further, let us introduce the following stability definition.

Definition 2. System (1) is said to be stochastically stable if for every initial condition $\phi(k) \in R^{n}, k=-d_{M},-d_{M}+1, \ldots, 0$ and $r_{0} \in \mathscr{I}$, the following holds:

$$
E\left\{\sum_{k=0}^{\infty}\|x(k)\|^{2} \mid \phi(\cdot), r_{0}\right\}<\infty
$$

\section{Main Results}

In this part, a new delay-dependent stochastic stability condition for MJLSs with partly known transition probabilities is presented in the following theorem.

Theorem 3. Consider the MJLSs (1) with partly known transition probabilities (3), if there exist symmetric matrices $P_{i}>$ $0, Q>0, R>0, Z_{1}>0, Z_{2}>0, M_{i v}, N_{i v}, S_{i v}, v=$ $1,2,3, i \in \mathscr{I}, l \in \mathscr{I}_{u k}^{i}$ such that

$$
\left[\begin{array}{cc}
E_{1}+E_{2}+E_{3} & E_{4} \\
* & E_{5}
\end{array}\right]<0
$$

where 


$$
\begin{aligned}
& \overline{\mathscr{P}}_{k}^{i}=\sum_{j \in \mathcal{F}_{k}^{i}} \bar{\pi}_{i j} P_{j}, \quad \underline{\pi}_{k}^{i}=\sum_{j \in \mathscr{P}_{k}^{i}} \underline{\pi}_{i j}, \\
& E_{1}=\left[\begin{array}{ccc}
A_{i}^{T}\left(\overline{\mathscr{P}}_{k}^{i}+\left(1-\underline{\pi}_{k}^{i}\right) P_{l}\right) A_{i}-P_{i}+\left(d_{M}-d_{m}+1\right) Q+R & A_{i}^{T}\left(\overline{\mathscr{P}}_{k}^{i}+\left(1-\underline{\pi}_{k}^{i}\right) P_{l}\right) A_{d i} & 0 \\
* & A_{d i}^{T}\left(\overline{\mathscr{P}}_{k}^{i}+\left(1-\underline{\pi}_{k}^{i}\right) P_{l}\right) A_{d i}-Q & 0 \\
* & * & -R
\end{array}\right] \text {, } \\
& E_{2}=\left[\begin{array}{lll}
M_{i}+N_{i} & S_{i}-M_{i}-N_{i}-S_{i}
\end{array}\right]+\left[\begin{array}{lll}
M_{i}+N_{i} & S_{i}-M_{i}-N_{i}-S_{i}
\end{array}\right]^{T} \text {, } \\
& E_{3}=d_{M}\left[\begin{array}{lll}
A_{i}-I & A_{d i} & 0
\end{array}\right]^{T}\left(Z_{1}+Z_{2}\right)\left[\begin{array}{lll}
A_{i}-I & A_{d i} & 0
\end{array}\right] \text {, } \\
& E_{4}=\left[\begin{array}{lll}
\sqrt{d_{M}} M_{i} & \sqrt{d_{r}} S_{i} & \sqrt{d_{M}} N_{i}
\end{array}\right] \text {, } \\
& E_{5}=\left[\begin{array}{ccc}
-Z_{1} & 0 & 0 \\
0 & -Z_{1} & 0 \\
0 & 0 & -Z_{2}
\end{array}\right] \text {. }
\end{aligned}
$$

Proof. Consider system (1) and construct a stochastic Lyapunov functional as

$$
V\left(x_{k}, r_{k}\right)=V\left(x_{k}, i\right)=\sum_{s=1}^{5} V_{s}\left(x_{k}, i\right), \quad i \in \mathscr{I},
$$

where

$$
\begin{aligned}
& V_{1}\left(x_{k}, i\right)=x_{k}^{T} P_{i} x_{k}, \\
& V_{2}\left(x_{k}, i\right)=\sum_{l=k-d_{k}}^{k-1} x^{T}(l) Q x(l), \\
& V_{3}\left(x_{k}, i\right)=\sum_{\theta=-d_{M}+1}^{-d_{m}} \sum_{l=k+\theta}^{k-1} x^{T}(l) Q x(l), \\
& V_{4}\left(x_{k}, i\right)=\sum_{l=k-d_{M}}^{k-1} x^{T}(l) R x(l), \\
& V_{5}\left(x_{k}, i\right)=\sum_{\theta=-d_{M}}^{-1} \sum_{l=k+\theta}^{k-1} y^{T}(l)\left(Z_{1}+Z_{2}\right) y(l), \\
& y(l)=x(l+1)-x(l), \quad d_{r}=d_{M}-d_{m} .
\end{aligned}
$$

Defining $\Delta V\left(x_{k}, i\right)=\sum_{s=1}^{5} \Delta V_{s}$, it follows that

$$
\begin{aligned}
\Delta V_{1}= & E\left[V_{1}\left(x_{k+1}, r_{k+1}=j \mid x_{k}, r_{k}=i\right)-V_{1}\left(x_{k}, r_{k}=i\right)\right] \\
= & x_{k+1}^{T} \sum_{j \in \mathscr{I}} \pi_{i j} P_{j} x_{k+1}-x_{k}^{T} P_{i} x_{k} \\
= & x_{k}^{T}\left(A_{i}^{T} \mathscr{P}_{i} A_{i}-P_{i}\right) x_{k}+2 x_{k}^{T} A_{i}^{T} \mathscr{P}_{i} A_{d i} x_{k-d(k)} \\
& +x_{k-d(k)}^{T} A_{d i}^{T} \mathscr{P}_{i} A_{d i} x_{k-d(k)},
\end{aligned}
$$

$$
\Delta V_{2}=E\left[V_{2}\left(x_{k+1}, r_{k+1}=j \mid x_{k}, r_{k}=i\right)-V_{2}\left(x_{k}, r_{k}=i\right)\right]
$$$$
=\left(\sum_{l=k+1-d_{k+1}}^{k}-\sum_{l=k-d_{k}}^{k-1}\right) x^{T}(l) Q x(l)
$$$$
=x_{k}^{T} Q x_{k}-x_{k-d_{k}}^{T} Q x_{k-d_{k}}+\sum_{l=k+1-d_{k+1}}^{k-d_{k}} x^{T}(l) Q x(l)
$$$$
\leq x_{k}^{T} Q x_{k}-x_{k-d_{k}}^{T} Q x_{k-d_{k}}+\sum_{l=k+1-d_{M}}^{k-d_{m}} x^{T}(l) Q x(l),
$$$$
\Delta V_{3}=E\left[V_{3}\left(x_{k+1}, r_{k+1}=j \mid x_{k}, r_{k}=i\right)-V_{3}\left(x_{k}, r_{k}=i\right)\right]
$$$$
=\sum_{\theta=-d_{M}+1}^{-d_{m}}\left(\sum_{l=k+1+\theta}^{k}-\sum_{l=k+\theta}^{k-1}\right) x^{T}(l) Q x(l)
$$$$
=\left(d_{M}-d_{m}\right) x_{k}^{T} Q x_{k}-\sum_{l=k+1-d_{M}}^{k-d_{m}} x^{T}(l) Q x(l),
$$$$
\Delta V_{4}=E\left[V_{4}\left(x_{k+1}, r_{k+1}=j \mid x_{k}, r_{k}=i\right)-V_{4}\left(x_{k}, r_{k}=i\right)\right]
$$$$
=\left(\sum_{l=k+1-d_{M}}^{k}-\sum_{l=k-d_{M}}^{k-1}\right) x^{T}(l) R x(l)
$$$$
=x_{k}^{T} R x_{k}-x_{k-d_{M}}^{T} R x_{k-d_{M}},
$$

$$
\begin{aligned}
& \Delta V_{5}= E\left[V_{5}\left(x_{k+1}, r_{k+1}=j \mid x_{k}, r_{k}=i\right)-V_{5}\left(x_{k}, r_{k}=i\right)\right] \\
&=\sum_{\theta=-d_{M}}^{-1}\left[\sum_{l=k+\theta+1}^{k} y^{T}(l)\left(Z_{1}+Z_{2}\right) y(l)\right. \\
&\left.-\sum_{l=k+\theta}^{k-1} y^{T}(l)\left(Z_{1}+Z_{2}\right) y(l)\right]
\end{aligned}
$$




$$
\begin{aligned}
= & \sum_{\theta=-d_{M}}^{-1}\left(\sum_{l=k+\theta+1}^{k}-\sum_{l=k+\theta}^{k-1}\right) y^{T}(l)\left(Z_{1}+Z_{2}\right) y(l) \\
= & \sum_{\theta=-d_{M}}^{-1}\left[y_{k}^{T}\left(Z_{1}+Z_{2}\right) y_{k}-y_{k+\theta}^{T}\left(Z_{1}+Z_{2}\right) y_{k+\theta}\right] \\
= & d_{M} y_{k}^{T}\left(Z_{1}+Z_{2}\right) y_{k}-\sum_{l=k-d_{k}}^{k-1} y^{T}(l) Z_{1} y(l) \\
& -\sum_{l=k-d_{M}}^{k-d_{k}-1} y^{T}(l) Z_{1} y(l)-\sum_{l=k-d_{M}}^{k-1} y^{T}(l) Z_{2} y(l) \\
= & d_{M} y_{k}^{T}\left(Z_{1}+Z_{2}\right) y_{k}-\sum_{l=k-d_{k}}^{k-1} y^{T}(l) Z_{1} y(l) \\
& -\sum_{l=k-d_{M}}^{k-d_{k}-1} y^{T}(l) Z_{1} y(l)-\sum_{k-d_{k}}^{k-1} y^{T}(l) Z_{2} y(l) \\
& -\sum_{k-d_{k}-1}^{k-d_{M}} y^{T}(l) Z_{2} y(l) .
\end{aligned}
$$

Summing up (10), one has

$$
\begin{aligned}
& \Delta V\left(x_{k}, r_{k}\right) \leq x_{k}^{T}\left(A_{i}^{T} \mathscr{P}_{i} A_{i}-P_{i}\right) x_{k}+2 x_{k}^{T} A_{i}^{T} \mathscr{P}_{i} A_{d i} x_{k-d_{k}} \\
& +x_{k-d_{k}}^{T} A_{d i}^{T} \mathscr{P}_{i} A_{d i} x_{k-d_{k}}-x_{k-d_{k}}^{T} Q x_{k-d_{k}} \\
& +\left(d_{M}-d_{m}+1\right) x_{k}^{T} Q x_{k}+x_{k}^{T} R x_{k} \\
& -x_{k-d_{M}}^{T} Q x_{k-d_{M}} \\
& +d_{M}\left[\left(A_{i}-I\right) x_{k}+A_{d i} x_{k-d_{k}}\right]^{T} \\
& \times\left(Z_{1}+Z_{2}\right)\left[\left(A_{i}-I\right) x_{k}+A_{d i} x_{k-d_{k}}\right] \\
& -\sum_{l=k-d_{k}}^{k-1} y^{T}(l) Z_{1} y(l) \\
& -\sum_{l=k-d_{M}}^{k-d_{k}-1} y^{T}(l) Z_{1} y(l)-\sum_{l=k-d_{k}}^{k-1} y^{T}(l) Z_{2} y(l) \\
& +2 \xi_{k}^{T} M_{i}\left[x_{k}-x_{k-d_{k}}-\sum_{l=k-d_{k}}^{k-1} y(l)\right] \\
& +2 \xi_{k}^{T} S_{i}\left[x_{k-d_{k}}-x_{k-d_{M}}-\sum_{l=k-d_{M}}^{k-d_{k}-1} y(l)\right] \\
& +2 \xi_{k}^{T} N_{i}\left[x_{k}-x_{k-d_{M}}-\sum_{l=k-d_{M}}^{k-1} y(l)\right] \text {. }
\end{aligned}
$$

Therefore, we have

$$
\begin{aligned}
\Delta V\left(x_{k}, r_{k}\right) \leq \xi^{T}(k)[ & \widehat{E}_{1}+E_{2}+E_{3}+d_{M} M_{i} Z_{1}^{-1} M_{i}^{T} \\
& +\left(d_{M}-d_{m}\right) \times S_{i} Z_{1}^{-1} S_{i}^{T} \\
& \left.+d_{M} N_{i} Z_{2}^{-1} N_{i}^{T}\right] \xi(k),
\end{aligned}
$$

where

$$
\begin{aligned}
& \xi(k)=\left[\begin{array}{lll}
x_{k}^{T} & x_{k-d_{k}}^{T} & x_{k-d_{M}}^{T}
\end{array}\right]^{T}, \\
& \widehat{E}_{1}=\left[\begin{array}{ccc}
A_{i}^{T}\left(\mathscr{P}_{i}\right) A_{i}-P_{i}+\left(d_{M}-d_{m}+1\right) Q+R & A_{i}^{T} \mathscr{P}_{i} A_{d i} & 0 \\
* & A_{d i}^{T} \mathscr{P}_{i} A_{d i}-Q & 0 \\
* & * & -R
\end{array}\right] \text {, } \\
& E_{2}=\left[M_{i}+N_{i} S_{i}-M_{i}-N_{i}-S_{i}\right] \\
& +\left[M_{i}+N_{i} S_{i}-M_{i}-N_{i}-S_{i}\right]^{T}, \\
& E_{3}=d_{M}\left[\begin{array}{lll}
A_{i}-I & A_{d i} & 0
\end{array}\right]^{T}\left(Z_{1}+Z_{2}\right)\left[\begin{array}{lll}
A_{i}-I & A_{d i} & 0
\end{array}\right], \\
& E_{4}=\left[\begin{array}{lll}
\sqrt{d_{M}} M_{i} & \sqrt{d_{r}} S_{i} & \sqrt{d_{M}} N_{i}
\end{array}\right] \text {, } \\
& E_{5}=\left[\begin{array}{ccc}
-Z_{1} & 0 & 0 \\
0 & -Z_{1} & 0 \\
0 & 0 & -Z_{2}
\end{array}\right] \\
& M_{i}=\left[\begin{array}{lll}
M_{i 1}^{T} & M_{i 2}^{T} & M_{i 3}^{T}
\end{array}\right]^{T} \text {, } \\
& N_{i}=\left[\begin{array}{lll}
N_{i 1}^{T} & N_{i 2}^{T} & N_{i 3}^{T}
\end{array}\right]^{T}, \\
& S_{i}=\left[\begin{array}{lll}
S_{i 1}^{T} & M_{i 2}^{T} & S_{i 3}^{T}
\end{array}\right]^{T} \text {. }
\end{aligned}
$$

On the other hand, the following formula holds:

$$
\mathscr{P}_{i}=\sum_{j \in \mathscr{J}} \pi_{i j} P_{j}=\sum_{j \in \mathscr{F}_{k}^{i}} \pi_{i j} P_{j}+\sum_{j \in \mathscr{F}_{u k}^{i}} \pi_{i j} P_{j} .
$$

Considering the fact that $\sum_{j=1}^{N} \pi_{i j}=1$, it yields

$$
\sum_{j \in \mathscr{F}_{k}^{i}} \pi_{i j}+\sum_{j \in \mathscr{F}_{u k}^{i}} \pi_{i j}=1 .
$$

Since transition probabilities are partly known, we have $\pi_{k}^{i}=$ $\sum_{j \in \mathscr{F}_{k}^{i}} \pi_{i j}<1$ and $\left(\sum_{j \in \mathscr{F}_{u k}^{i}} \pi_{i j}\right) /\left(1-\pi_{k}^{i}\right)=1$. Therefore, it follows that

$$
\begin{aligned}
A_{i}^{T} \mathscr{P}_{i} A_{i} & =A_{i}^{T}\left(\sum_{j \in I_{k}^{i}} \pi_{i j} P_{j}+\sum_{j \in I_{u k}^{i}} \pi_{i j} P_{j}\right) A_{i} \\
& =A_{i}^{T}\left(\mathscr{P}_{k}^{i}+\sum_{j \in I_{u k}^{i}} \pi_{i j} P_{j}\right) A_{i} \\
& =A_{i}^{T}\left(\mathscr{P}_{k}^{i}+\left(1-\pi_{k}^{i}\right) \sum_{j \in I_{u k}^{i}} \frac{\pi_{i j}}{1-\pi_{k}^{i}} P_{j}\right) A_{i} \\
& =\sum_{j \in I_{u k}^{i}} \frac{\pi_{i j}}{1-\pi_{k}^{i}}\left[A_{i}^{T}\left(\mathscr{P}_{k}^{i}+\left(1-\pi_{k}^{i}\right) P_{j}\right) A_{i}\right] .
\end{aligned}
$$

According to (6), we can get $\Delta V\left(x_{k}, r_{k}\right)<0$. 
Remark 4. According to Theorem 3, when calculating the forward difference of $\Delta V_{5},-\sum_{l=k-d_{M}}^{k-1} y^{T}(l) Z_{1} y(l)$ is divided as $-\sum_{-k-d_{k}}^{k-1} y^{T}(l) Z_{1} y(l)$ and $-\sum_{-k-d_{M}}^{k-d_{k}-1} y^{T}(l) Z_{1} y(l)$.

Remark 5. Different from the existing result [32], to separate Lyapunov variables from unknown transition probabilities, the property of transition probabilities is made full use of, namely, $\sum_{j \in \mathcal{F}_{u k}^{i}} \pi_{i j}=1-\sum_{j \in \mathscr{F}_{k}^{i}} \pi_{i j}$. Thanks to this separation, the method proposed in Theorem 3 is less conservative than that of [32].

\section{Illustrative Example}

To show the effectiveness of the proposed method, a numerical example is given below.

Example 1. Consider the MJLSs (1) with four operation modes and the following system matrices:

$$
\begin{aligned}
A_{1} & =\left[\begin{array}{cc}
2 & 0.56 \\
0.25 & 1
\end{array}\right], & A_{2} & =\left[\begin{array}{cc}
7 & 0.64 \\
0 & -0.24
\end{array}\right], \\
A_{3} & =\left[\begin{array}{cc}
-1.23 & 0.65 \\
0.44 & -0.87
\end{array}\right], & A_{4} & =\left[\begin{array}{cc}
4 & 0.64 \\
0.26 & 5
\end{array}\right], \\
A_{d 1} & =\left[\begin{array}{cc}
-0.03 & 0.11 \\
0 & 0.13
\end{array}\right], & A_{d 2} & =\left[\begin{array}{cc}
0.13 & 0 \\
0.02 & 0.03
\end{array}\right], \\
A_{d 3} & =\left[\begin{array}{cc}
0.02 & -0.12 \\
0.07 & -0.14
\end{array}\right], & A_{d 4} & =\left[\begin{array}{cc}
0.9 & 0.07 \\
0.04 & -0.05
\end{array}\right] .
\end{aligned}
$$

Our purpose is to check the stability of (1) with different cases of transition probabilities.

Case 1. Transition probabilities are known

$$
\left[\begin{array}{cccc}
0.4 & 0.6 & 0 & 0 \\
0.2 & 0.6 & 0.19 & 0.01 \\
0.2 & 0.3 & 0.4 & 0.1 \\
0.3 & 0.3 & 0.3 & 0.1
\end{array}\right] .
$$

In this case, given $d_{M}=0$ and $d_{m}=0$, system (1) is unstable even if all the transition probabilities are known. It implies that the underlying system will be unstable for any timevarying delay starting from $d_{m}=0$.

Case 2. Transition probabilities are partly known

$$
\left[\begin{array}{cccc}
0.4 & 0.6 & 0 & 0 \\
0.2 & 0.6 & 0.19 & 0.01 \\
? & 0.3 & ? & 0.1 \\
0.3 & ? & 0.3 & ?
\end{array}\right]
$$

In this case, we assume that the range of the time-varying delay belongs to [ 110$]$. By solving the conditions given in Theorem 1 of [32] and Theorem 3 in this paper, respectively, no feasible solution can be found for the former. Therefore, the method proposed in this paper is less conservative than the existing result.
TABLE 1

\begin{tabular}{lccc}
\hline TPs & Case 1 & Case 2 & Case 3 \\
\hline$d(k)$ & $1 \leq d(k) \leq 20$ & $1 \leq d(k) \leq 10$ & $1 \leq d(k) \leq 5$ \\
Theorem 3 & Feasible & Feasible & Feasible \\
\hline
\end{tabular}

Case 3. We consider the case that there are unknown elements in the transition probability matrix varying an interval

$$
\left[\begin{array}{cccc}
0.4 & 0.6 & 0 & 0 \\
0.2 & \alpha & 0.19 & ? \\
? & 0.3 & ? & 0.1 \\
0.3 & ? & 0.3 & ?
\end{array}\right]
$$

where $0.1 \leq \alpha \leq 0.4$.

By solving the conditions given in Theorem 3, Table 1 is obtained.

According to Table 1, it can be seen that the more transition probabilities knowledge we have, the larger the delay range can be obtained.

\section{Conclusions}

The stability analysis for a class of discrete-time MJLSs with time-varying delay and partly known transition probabilities is investigated in this paper. To separate Lyapunov variables from unknown transition probabilities, the property of transition probabilities is made full use of. Via constructing an appropriate Lyapunov function, a novel stability criterion is obtained in the framework of linear matrix inequality. The effectiveness of the proposed approach is demonstrated by a numerical example. In the future, the obtained results are considered to be extended to deal with the fault tolerant control of Markov jump systems with partly known transition probabilities and mixed time-varying delay.

\section{Conflict of Interests}

The authors declare that there is no conflict of interests regarding the publication of this paper.

\section{Acknowledgments}

This work is supported by the National Natural Science Foundation of China (Grants nos. 61273355, 61273356, and 61035005), the Foundation of State Key Laboratory of Robotics (Grant no. Z2013-06), and Natural Science Foundation of Liaoning Province, China (Grant no. 2014020082).

\section{References}

[1] O. L. Costa, M. D. Fragoso, and R. P. Marques, Discrete-Time Markov Jump Linear Systems, Springer, London, UK, 2005.

[2] C. E. de Souza, A. Trofino, and K. A. Barbosa, "Modeindependent $H_{\infty}$ filters for Markovian jump linear systems," IEEE Transactions on Automatic Control, vol. 51, no. 11, pp. 18371841, 2006. 
[3] C. E. de Souza, "Robust stability and stabilization of uncertain discrete-time Markovian jump linear systems," IEEE Transactions on Automatic Control, vol. 51, no. 5, pp. 836-841, 2006.

[4] A. P. Goncalves, A. R. Fioravanti, and J. C. Geromel, " $H_{\infty}$ filtering of discrete-time Markov jump linear systems through linear matrix inequalities," IEEE Transactions on Automatic Control, vol. 54, no. 6, pp. 1347-1351, 2009.

[5] Q. Zhu, "pth moment exponential stability of impulsive stochastic functional differential equations with Markovian switching," Journal of the Franklin Institute, vol. 351, no. 7, pp. 3965-3986, 2014.

[6] D. Li and Q. Zhu, "Comparison principle and stability of stochastic delayed neural networks with Markovian switching," Neurocomputing, vol. 123, pp. 436-442, 2014.

[7] K. Benjelloun and E. K. Boukas, "Mean square stochastic stability of linear time-delay system with Markovian jumping parameters," IEEE Transactions on Automatic Control, vol. 43, no. 10, pp. 1456-1460, 1998.

[8] H. Fujisaki, "On correlation values of $M$-phase spreading sequences of Markov chains," IEEE Transactions on Circuits and Systems I: Fundamental Theory and Applications, vol. 49, no. 12, pp. 1745-1750, 2002.

[9] D. Ye and G. H. Yang, "Delay-dependent adaptive reliable $H_{\infty}$ control of linear time-varying delay systems," International Journal of Robust and Nonlinear Control, vol. 19, no. 4, pp. 462479, 2009.

[10] D. Ye and G.-H. Yang, "Robust switching-type $H_{\infty}$ filter design for linear uncertain systems with time-varying delay," Information Sciences, vol. 181, no. 9, pp. 1686-1699, 2011.

[11] H. Gao and T. Chen, "New results on stability of discrete-time systems with time-varying state delay," IEEE Transactions on Automatic Control, vol. 52, no. 2, pp. 328-334, 2007.

[12] S. Xu and J. Lam, "Improved delay-dependent stability criteria for time-delay systems," IEEE Transactions on Automatic Control, vol. 50, no. 3, pp. 384-387, 2005.

[13] Y. He, Q. Wang, C. Lin, and M. Wu, "Delay-range-dependent stability for systems with time-varying delay," Automatica, vol. 43, no. 2, pp. 371-376, 2007.

[14] E. K. Boukas, Z. K. Liu, and P. Shi, "Delay-dependent stability and output feedback stabilisation of Markov jump system with time-delay," IEE Proceedings: Control Theory and Applications, vol. 149, no. 5, pp. 379-386, 2002.

[15] W.-H. Chen, J.-X. Xu, and Z.-H. Guan, "Guaranteed cost control for uncertain Markovian jump systems with mode-dependent time-delays," IEEE Transactions on Automatic Control, vol. 48, no. 12, pp. 2270-2277, 2003.

[16] Y.-Y. Cao, J. Lam, and L. Hu, "Delay-dependent stochastic stability and $H_{\infty}$ analysis for time-delay systems with Markovian jumping parameters," Journal of the Franklin Institute. Engineering and Applied Mathematics, vol. 340, no. 6-7, pp. 423434, 2003.

[17] J. Wu, T. W. Chen, and L. Wang, "Delay-dependent robust stability and $H_{\infty}$ control for jump linear systems with delays," Systems \& Control Letters, vol. 55, no. 11, pp. 939-948, 2006.

[18] S. Xu, J. Lam, and X. Mao, "Delay-dependent $H_{\infty}$ control and filtering for uncertain Markovian jump systems with timevarying delays," IEEE Transactions on Circuits and Systems. I. Regular Papers, vol. 54, no. 9, pp. 2070-2077, 2007.

[19] J. Wang and Y. Luo, "Further improvement of delay-dependent stability for Markov jump systems with time-varying delay," in Proceedings of the 7th IEEE World Congress on Intelligent Control and Automation (WCICA '08), pp. 6319-6324, Chongqing, China, June 2008.

[20] X. D. Zhao and Q. S. Zeng, "Delay-dependent stability analysis for Markovian jump systems with interval time-varying-delays," International Journal of Automation and Computing, vol. 7, no. 2, pp. 224-229, 2010.

[21] Z.-G. Wu, P. Shi, H. Su, and J. Chu, "Stochastic synchronization of markovian jump neural networks with time-varying delay using sampled data," IEEE Transactions on Cybernetics, vol. 43, no. 6, pp. 1796-1806, 2013.

[22] R. Rakkiyappan, Q. Zhu, and T. Radhika, "Design of sampled data state estimator for Markovian jumping neural networks with leakage time-varying delays and discontinuous Lyapunov functional approach," Nonlinear Dynamics, vol. 73, no. 3, pp. 1367-1383, 2013.

[23] Q. Zhu and J. Cao, "Robust exponential stability of markovian jump impulsive stochastic Cohen-Grossberg neural networks with mixed time delays," IEEE Transactions on Neural Networks, vol. 21, no. 8, pp. 1314-1325, 2010.

[24] Q. Zhu and J. Cao, "Stability analysis of markovian jump stochastic BAM neural networks with impulse control and mixed time delays," IEEE Transactions on Neural Networks and Learning Systems, vol. 23, no. 3, pp. 467-479, 2012.

[25] Q. Zhu and J. Cao, "Exponential stability of stochastic neural networks with both Markovian jump parameters and mixed time delays," IEEE Transactions on Systems, Man, and Cybernetics B: Cybernetics, vol. 41, no. 2, pp. 341-353, 2011.

[26] Q. Zhu and J. Cao, "Stability of Markovian jump neural networks with impulse control and time varying delays," Nonlinear Analysis: Real World Applications, vol. 13, no. 5, pp. 2259-2270, 2012.

[27] Q. Zhu, F. Xi, and X. Li, "Robust exponential stability of stochastically nonlinear jump systems with mixed time delays," Journal of Optimization Theory and Applications, vol. 154, no. 1, pp. 154-174, 2012.

[28] L. Zhang and E.-K. Boukas, "Mode-dependent filtering for discrete-time Markovian jump linear systems with partly unknown transition probabilities," Automatica, vol. 45, no. 6, pp. 1462-1467, 2009.

[29] L. Zhang and J. Lam, "Necessary and sufficient conditions for analysis and synthesis of Markov jump linear systems with incomplete transition descriptions," IEEE Transactions on Automatic Control, vol. 55, no. 7, pp. 1695-1701, 2010.

[30] L. Zhang, E.-K. Boukas, and P. Shi, " $H_{\infty}$ model reduction for discrete-time Markov jump linear systems with partially known transition probabilities," International Journal of Control, vol. 82, no. 2, pp. 343-351, 2009.

[31] M. Shen, G. Zhang, Y. Yuan, and D. Ye, "Relaxed $H_{\infty}$ controller design for continuous Markov jump system with incomplete transition probabilities," Circuits, Systems, and Signal Processing, vol. 33, no. 5, pp. 1393-1410, 2014.

[32] L. Zhang, E.-K. Boukas, and J. Lam, "Analysis and synthesis of Markov jump linear systems with time-varying delays and partially known transition probabilities," IEEE Transactions on Automatic Control, vol. 53, no. 10, pp. 2458-2464, 2008. 


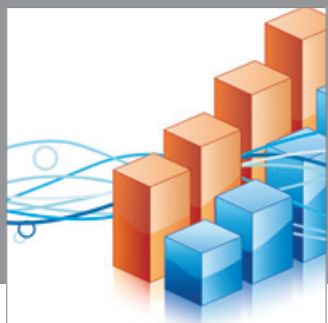

Advances in

Operations Research

mansans

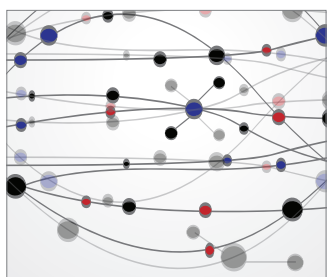

The Scientific World Journal
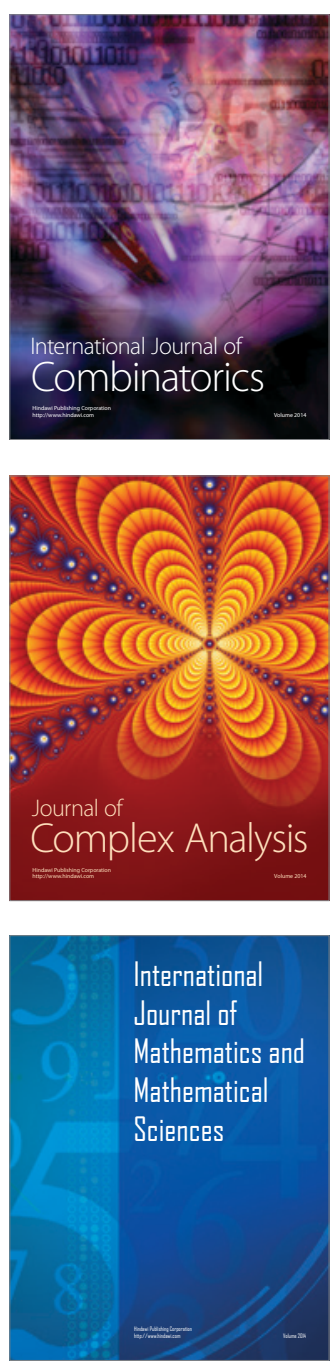
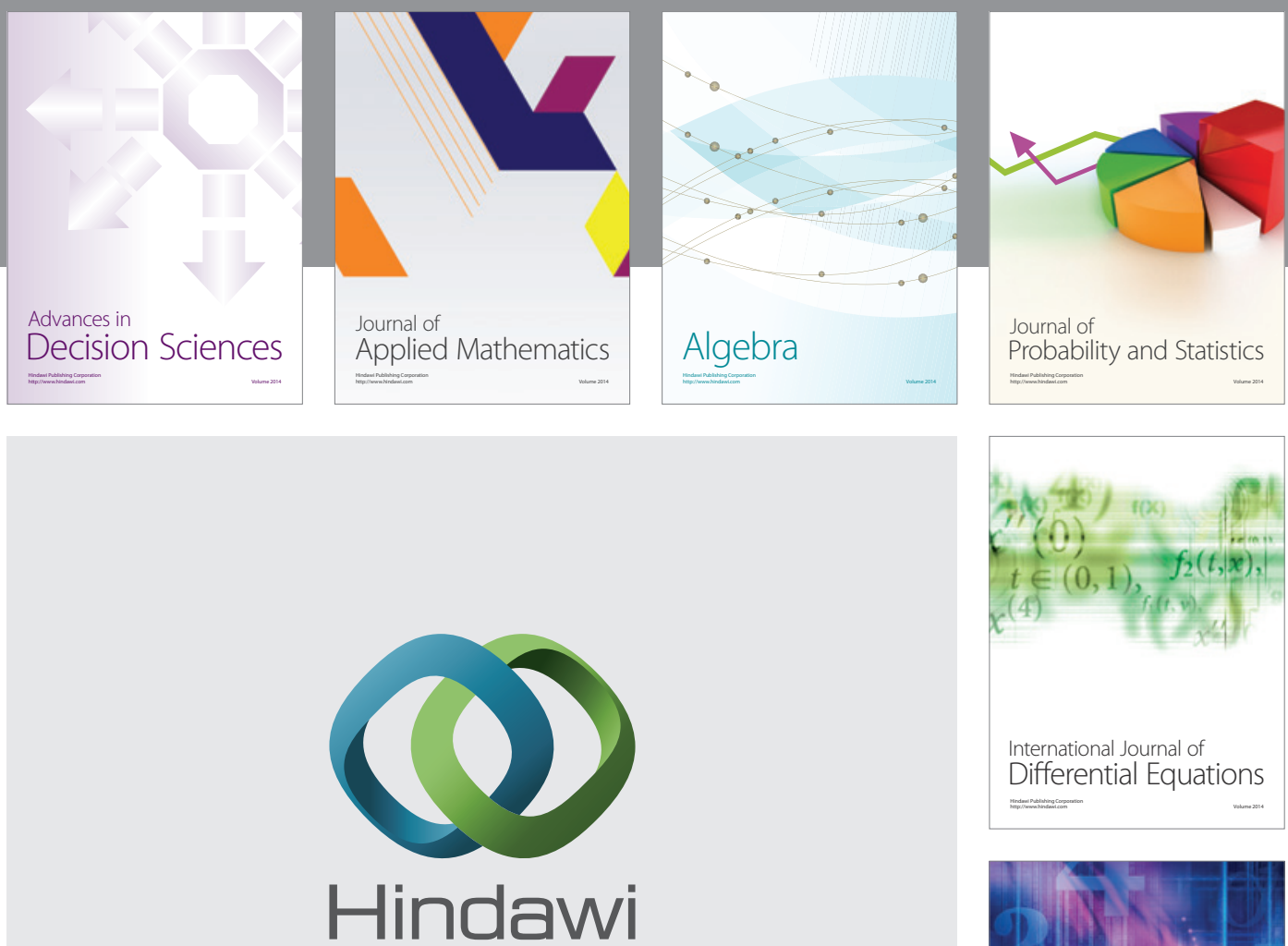

Submit your manuscripts at http://www.hindawi.com
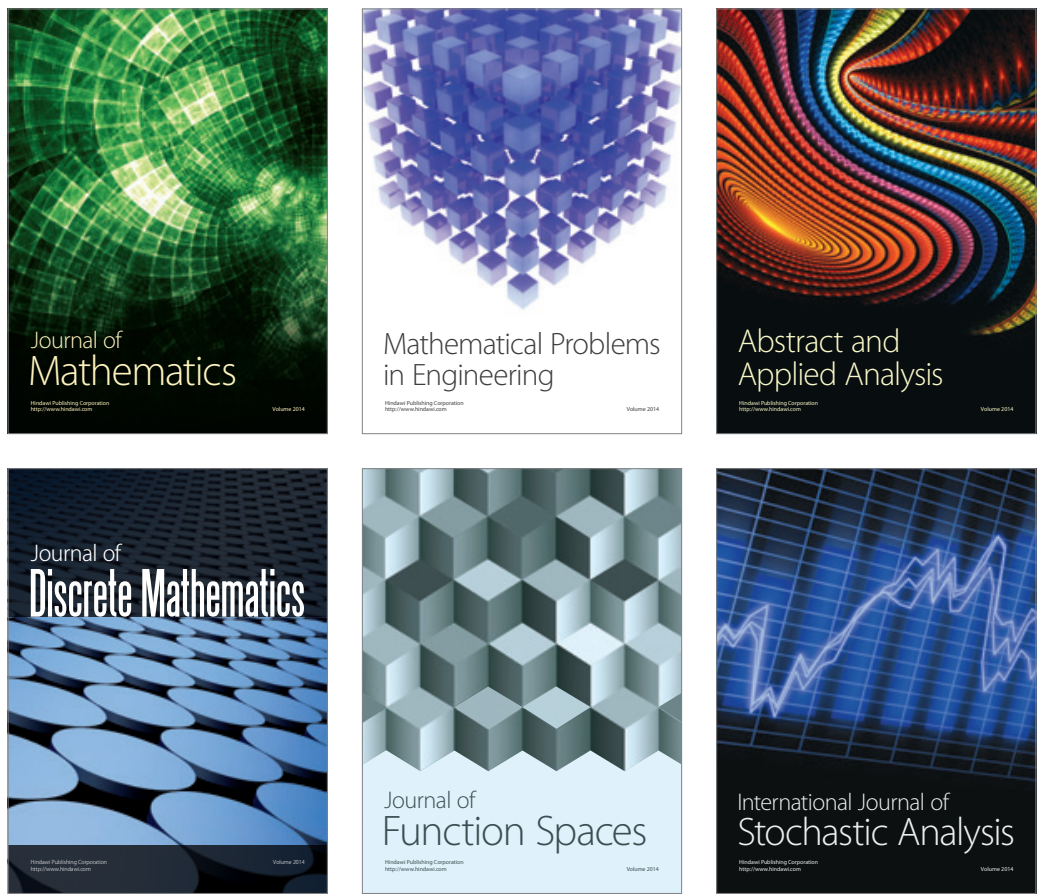

Journal of

Function Spaces

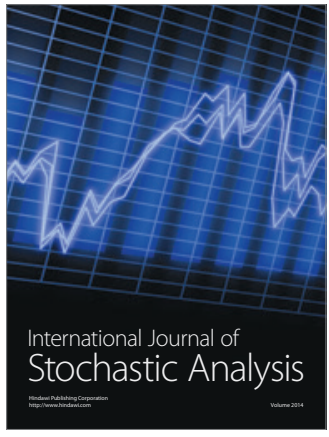

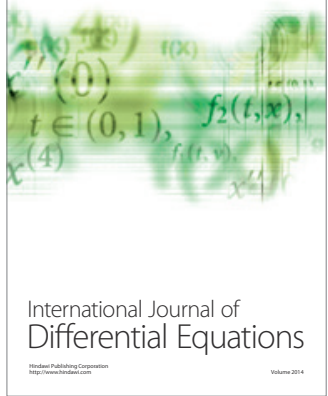
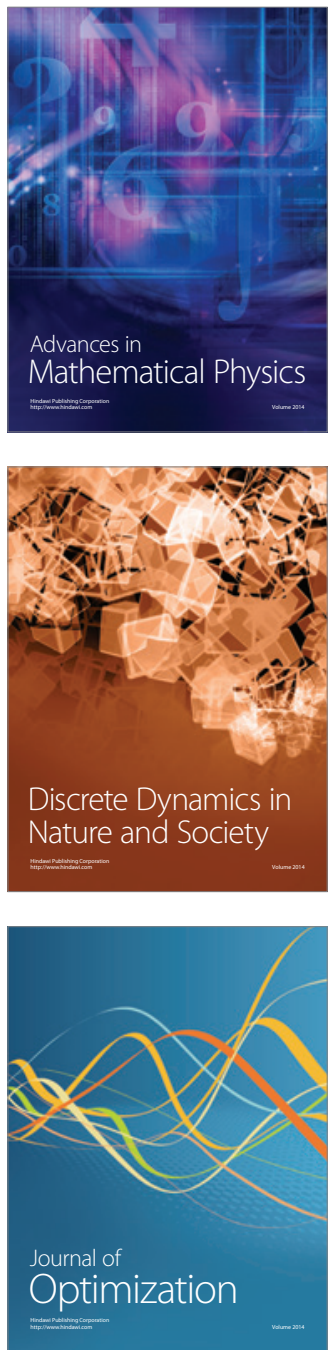